\title{
Synthesis and Characterization of Chitosan/Gum Arabic Nanoparticles for Bone Regeneration
}

\author{
Chinenye Appolonia Ibekwe ${ }^{1, *}$, Grace Modupe Oyatogun ${ }^{1}$, Temitope Ayodeji Esan², Kunle Michael Oluwasegun ${ }^{1}$ \\ ${ }^{1}$ Department of Materials Science and Engineering, Obafemi Awolowo University, Ile-Ife, Nigeria \\ ${ }^{2}$ Department of Restorative Dentistry, Faculty of Dentistry, Obafemi Awolowo University, Ile-Ife, Nigeria \\ *Corresponding author: chibek2020@yahoo.com
}

\begin{abstract}
Chitosan /gum arabic nanoparticles (C/G)have been prepared by ionic gelation method. This was with a view to enhance the mechanical properties and its application as bone graft scaffold. The cowry shells were washed, dried, pulverized and subsequently sieved with mesh No. 60, size $250 \mu \mathrm{m}$. It was deproteinized, Chitin was isolated from the synthesis by demineralising in $0.5 \mathrm{M}$ Hydrochloric acid, and subsequently deacetylated by the addition of $40 \%(\mathrm{~W} / \mathrm{V})$ of Sodium hydroxide to synthesize chitosan. The raw chitosan was purified using $2 \%$ (v/v) acetic acid solution. The synthesized chitosan and gum arabic, a product of Acacia tree, were used to prepare chitosan/gum arabic nanoparticles by ionic gelation method. Mechanical characterization was carried out on the synthesized material using universal testing machine. Analysis of the chemical composition was carried out using Fourier transform infrared spectrometer (FTIR) and X-Ray fluorescence, (XRF). Furthermore, the morphology of the materials were studied using scanning electron microscopy, SEM and the dimension of the nanoparticles were characterized using transmission electron microscopy (TEM). Finally, an attempt was made to ascertain its suitability for bone regeneration. The FTIR spectra result confirmed that the nanoparticle was actually a derivative of chitosan by the observed shift in the peak 3462 to $3404 \mathrm{~cm}^{-1}$. There is presence of a new peak at $1636 \mathrm{~cm}^{-1}$ and $1473 \mathrm{~cm}^{-1}$. Peak observed at $1080 \mathrm{~cm}^{-1}, 860 \mathrm{~cm}^{-1}$ and $712 \mathrm{~cm}^{-1}$ on $\mathrm{C} / \mathrm{G}$ nanoparticles spectrum were similar to the native chitosan spectrum which shows that there was no change in the main backbone of chitosan structure. The scanning electron microscopy (SEM) study revealed chitosan as polymeric rods, while the chitosan /gum arabic nanoparticles in aggregate. The TEM was to confirm nanoparticles of average size of 200nm. The ultimate compressive strength was found to have increased by $78.21 \%$, the Young Modulus by $54.4 \%$ and percentage elongation by $7 \%$. In overall assessment, mechanical properties of the chitosan/gum arabic nanoparticles were better than native chitosan. The study concluded that crosslinking of chitosan with gum arabic to form its nanoparticles derivative improved the mechanical properties of chitosan and consequently its application as a bone graft substitute for bone regeneration.
\end{abstract}

Keywords: Chitosan, gum arabic, nanoparticles; bone graft scaffold, bone regeneration.

Cite This Article: Chinenye Appolonia Ibekwe, Grace Modupe Oyatogun Temitope Ayodeji Esan, and Kunle Michael Oluwasegun, "Synthesis and Characterization of Chitosan/Gum Arabic Nanoparticles for Bone Regeneration” American Journal of Materials Science and Engineering, vol. 5, no. 1 (2017): 28-36. doi: 10.12691/ajmse-5-1-4.

\section{Introduction}

Most often, bone disease and injuries such as osteoarthritis, rheumatoid arthritis, osteoporosis and bone fracture, dramatically affect the quality of life of patients and limit their ability to perfume basic tasks such as walking, running and standing. The high occurrence of these conditions are evidence by more than 2.2 million bone graft used in orthopaedic annually worldwide [1]. Bone grafts are used to augments or stimulates the formation of new bone, in cases such as the healing of the skeletal fractures, or between bones across a diseased joint; to replace and regenerate lost bone as a result of trauma, infection, or disease or to improve bone healing response and regeneration of tissues around surgical implanted devices. The three main types of bone grafts are autograft, allograft and xenograft. The tissue regeneration of these bone graft is measured in terms of their osteogenic, osteoconductive and osteoinductive potential [2]. Autografts are considered the gold standard for bone repair; however, such as bone donor site morbidity $([1,3])$. Another alternative is the allograft, which is bone tissue from cadaver or living donors, eliminates donor site morbidity, but has the potential risk of adverse immunological response and also lacks osteogenic capacity of autograft [4]. Xenograft, which is bone graft from a species other than human such as bovine, constitutes another alternative for bone repair. This has its limitation in that it elicits cytotoxine effects which may lead to adverse immunological response [5]. The limitations associated with the utilization of these bone grafts have prompted the search for other alternatives, which is referred to as bone graft substitutes.

Bone graft substitute is based on the concept of bone tissue engineering, which aims at improving the 
osteoconductive, osteogenic and osteoinductive potential by incorporating bone progenitor cells and growth factors to scaffold made of various natural and synthetic materials, or combination, in order to mimic the bone microenvironment [3]. Tissue engineering is a helpful alternative strategy for conventional treatment in medicine. It was officially coined at a National Science Foundation Workshop in 1988 to mean "the application of principles and methods of engineering and life science toward the fundamental understanding of structure-function relationships in normal and pathological mammalian tissues and the development of biological substitutes to restore, maintain or improve the tissue function or the whole organs" [6]. The field relies on the use of threedimensional scaffolds to provide appropriate environment for the regeneration of tissues and organs $[1,6]$.

Scaffolds provide three dimensional surfaces for cell seeding, proliferation and filling of bone defects. It also provides mechanical competence during bone regeneration. The selection of the most suitable material for the production of bone regeneration scaffold is a determinant step, since its properties will determine the final attributes of the regenerated bone. Osteoconductivity, porosity and biodegradability are some of the required properties needed for scaffolds to be successful in bone tissue engineering. These properties will enhance bone formation, angiogenesis, support the attachment and proliferation of osteoblasts cells on the scaffold [7].

Polymeric biomaterials, which are biocompatible with the physiological system, have found wide applications in regenerative medicine and tissue engineering [1]. Biodegradable polymers are appropriate substrates for cells to attach, grow and maintain a differentiated phenotype [7]. They have also attracted significant interest because of their flexibility, in terms of chemical manipulation, and their biodegradability [3]. Natural materials, due to their bioactive have been observed to influence cell morphology, modulation, and differentiation [1]. These properties tend to have better interaction with cells. This allows them to have better performance in service. Natural polymer can be classified as proteins such as silk, collagen, fibrinogen, elastin and myosin, polysaccharides such as cellulose, amylose, dextran, chitin, chitosan and glycosaminoglycan, or polynucleotides, deoxyribonucleic acid, DNA, and ribonucleic acid, RNA [8].

Chitosan is a co-polymer of $\mathrm{N}$-acetylglucosamine and $\mathrm{N}$-glucosamine units, which are produced by alkaline deacetylation of naturally occurring chitin. It presents excellent biological properties such as biocompatibility, biodegradability and immunogenicity antibacterial activity, wound healing properties and bio-adhesive character. These attributes enhanced its biomedical applications [1,9]. It is isolated from exoskeleton of insects, arthropods and crustaceans (crabs lobsters, and shrimps). Internal shells of cephalopods, radulae of molluscs, scales of fish and lissamphibians are alternative sources of chitin [10]. Chitosan is a natural biological polymer possessing reactive amine and hydroxyl group that promotes osteoblast growth and in-vivo bone formation [9]. The molecular weight of chitosan may range from 300 to $1000 \mathrm{KDa}$, depending on its origin and the method of preparation. Chitosan is semi crystalline polymer and its crystallization depends on the degree of deacetylation [1]
The solubility, biodegradability, reactivity, and absorption of its substrates depend on the amount of protonated amino groups in the polymer chain [1,9] The cationic nature of chitosan allows electrostatic interaction with anionic glycosaminoglycan and proteoglycans [1].

Chitosan has found diverse applications in regenerative medicine, orthopedics, periodontology, drug delivery systems, wound healing and tissue engineering $[9,10]$. However, chitosan low mechanical resistance limits its application in tissue engineering. In other to optimize resistance and elasticity, crosslinking agents such polyethyleneglycol, dialdehydes (such as glutaraldehyde and glyoxal), and starch are used [9]. Chitosan as natural polymer finds immense application in various fields. Despite the intensive research on the transformation of chitosan to novel biomaterial, still research for suitable cross linkers to have high mechanical strength chitosan based scaffold material is ongoing [11]. The work, therefore exploit the dual role of gum arabic for the preparation of chitosan based scaffold with enhanced mechanical properties.

Gum arabic (GA) is a natural complex mixture of hydrophilic carbohydrate and hydrophobic protein component obtained from the stems and branches of Acacia Senegal $[12,13]$. GA consists mainly of high-molecular weight polysaccharides and their calcium, magnesium and potassium salts, which on hydrolysis yield arabinose, galactose, rhamnose and glucuronic acid. It is highly soluble in water to form a solution of $\mathrm{pH}$ - 4.5 and insoluble in alcohol [12,14]. Gum arabic has diverse industrial applications such as acting as a stabilizer, thickening agent and emulsifier in food industry as well as textile, lithography, cosmetics and pharmaceutical industries [14]. GA has also found wide applications in nanotechnology, where it has been used as a crosslinking agent to produce chitosan/gum arabic nanoparticles for sustained drug release [15].

Nanoparticles are defined as particulate dispersions or solid particles with a size in the range of 1-1000 nm $[16,17]$. The physical and biological properties of nanomaterials are unique and differ depending on their corresponding bulk material, making these entities an intense focus of recent studies. Nanostructured surfaces play major role in advanced biomedical implant design because of their enhanced bioactive properties, as well as their incompatible behavior toward bacterial colonization [18]. Several attempts have been made to synthesize chitosan nanoparticles from different chitosan sources. Different methods, such as the microemulsion method, reverse micellar method, self-assembling method and ionic gelation have been used to prepare chitosan nanoparticles [16,19].

Ionotropic gelation method involves the formation of chitosan nanoparticles based on the electrostatic interaction between the amine group of chitosan and negatively charged group of polyanions [19]. The mechanism of chitosan nanoparticle formation is based on the electrostatic interaction between amine group of chitosan and negatively charged group of polyanion such as tripolyphosphate. This technique offers a simple and mild preparation method in the aqueous environment [16,19]. Consequently, this method was adopted in this work to synthesize cowry shell based chitosan /gum arabic nanoparticles for bone regeneration. 


\section{Materials and Methods}

\subsection{Materials}

Materials used for this work were cowry shells and gum arabic were gotten from Il-Ife Market in Nigeria, and reagent and chemical such as hydrochloric acid, acetic acid, sodium hydroxide and acetone are from BDH Chemicals Ltd Poole, England and Sigma-Aldrich Laborchemickalien GMBH, Seelze, Germany. Distilled water was the solvent used throughout the work.

\subsection{Synthesis of Chitosan from Cowry Shell}

The cowry shells were washed with distilled water, dried, pulverized and subsequently sieved with standard mesh No. 60, size $250 \mu \mathrm{m}$. The pulverized cowry shells were put in a conical flask and $0.1 \mathrm{M} \mathrm{NaOH}$ was added. The mixture was boiled and stirred at $100^{\circ} \mathrm{C}$ for 2 hours in a water bath. The product was then filtered and washed with distilled water. Red litmus paper was used to check whether the filtrate $\mathrm{pH}$ was completely neutral. It was then dried in an oven at a temperature of $80^{\circ} \mathrm{C}$. The deproteinized product was decolorized by soaking in pure acetone for 24 hours and was subsequently washed and dried in an oven at $80^{\circ} \mathrm{C}$. Then, $2250 \mathrm{ml}$ of hydrochloric acid $(0.5 \mathrm{M})$ was added to the deproteinized cowry shell (500 g) to leach out $\mathrm{CaCO}_{3}$ and the mixture was boiled and stirred for 45 minutes at $100^{\circ} \mathrm{C}$ in a water bath and subsequent washing and drying were done. The residue, chitin was dried in an oven at $100^{\circ} \mathrm{C}$ for 2 hours. Furthermore, the chitin was boiled in $40 \%$ (W/V) of sodium hydroxide, at $100^{\circ} \mathrm{C}$ for 3 hours in water bath and it was cooled for 30minutes at room temperature to convert the chitin to chitosan. The product was filtered and the filtrate, chitosan, was washed and tested with litmus paper until the $\mathrm{pH}$ was neutral. The chitosan was subsequently dried overnight in an oven at a low temperature $25^{\circ} \mathrm{C}$. Furthermore, the raw chitosan was dissolved in $2 \%(\mathrm{v} / \mathrm{v})$ acetic acid solution and filtered to remove the residue of insoluble particles. Afterwards $2 \mathrm{M}$ of $\mathrm{NaOH}$ was added to the filtrate to obtain chitosan in form of white precipitate. The precipitated chitosan was washed thoroughly using distilled water, and dried at $40^{\circ} \mathrm{C}$ for 48 hours [20].

\subsection{Preparation of Nanoparticles}

Chitosan nanoparticles were prepared using the ionotropic gelation method used by $[19,20]$ with these steps. The use of acetic acid to dissolve chitosan was avoided due to the fact that chitosan can protonate in presence of gum arabic. Purified chitosan $(1.5 \% \mathrm{w} / \mathrm{v})$ was dispersed in distilled water. The gum arabic (1\% w/v) was dissolving in distilled water to obtain a known concentration under magnetic stirring at room temperature. Then $5 \mathrm{ml}$ of gum arabic solution (1\% w/v) was added into the chitosan $(5 \mathrm{ml})$ through an insulin syringe needle at the speed of $60 \mathrm{ml} / \mathrm{h}$ under magnetic stirring at room temperature. Equation (1)

$$
\begin{aligned}
& \mathrm{RNH}_{2}+\mathrm{R}^{{ }^{\top} \mathrm{COOH}} \rightarrow \mathrm{RN}^{+} \mathrm{H}_{3}+\mathrm{R}^{{ }^{\prime} \mathrm{COO}-} \\
& \quad \rightarrow \mathrm{RNH}_{3} \mathrm{OCR}^{\prime}+\mathrm{H}_{2} \mathrm{O} .
\end{aligned}
$$

The resulting chitosan nanoparticle suspension was centrifuged at $3000 \mathrm{rpm}$ for 20 minutes. Supernatant was discarded and nanoparticles were resuspended with distilled water. The samples were dried by air flow at $25^{\circ} \mathrm{C}$.

\subsection{Characterization of Chitosan and Chitosan/gum Arabic Nanoparticles Synthesized}

The compressive tests were performed on test samples. The particles were compressed to round shaped test samples of diameter and thickness $5.69 \mathrm{~mm}$ and $13.00 \mathrm{~mm}$ respectively with an aluminum mold. Compressive tests were carried out on the test samples with the aid of a Computerized Mechanical Testing Machine (Instron machine series 3369). The samples were subjected to axial compressive load at a crosshead speed of $2 \mathrm{~mm} / \mathrm{min}$. Load and displacement data were used to determine the compressive strength, relative deformation, modulus of elasticity and yield strength for each sample.

Chemical analyses of the nanoparticles were carried out using FTIR (FTIR 410® Jasco Colchester, United Kingdom) and XRF techniques .was carried out on chitosan extracted from cowry shell, gum arabic and synthesized chitosan/gum arabic nanoparticles. The surface of chitosan/gum arabic nanoparticles, chitosan and gum arabic were analyzed to determine their chemical composition. About $0.5 \mathrm{~g}$ of the test samples were pelletized each to obtain cylindrical pellets suitable for the XRF machine model HERZOG PW1606. Each of the pellets were mounted on the sample holder and each sample were irradiated for 20 minutes at a fixed tube operating condition of $25 \mathrm{kVA}$ and $6 \mathrm{~mA}$. The test result was displayed on the computer connected to the XRF and the percentage of the elements present were analyzed and recorded.

The chemical interaction between the chitosan and gum arabic molecules were established using FTIR spectrometry. Five milligrams of the analyst were mixed thoroughly with about $100 \mathrm{mg}$ of dry $\mathrm{KBr}$ and appropriate amounts of the disk were prepared by compression to form $\mathrm{KBr}$ pellets for the test. All samples were scanned in the wave number region from 4000 and $400 \mathrm{~cm}^{-1}$. The

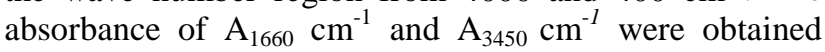
from chitosan spectrum and used to calculate the degree of deacetylation as shown in equation (2).

$$
D D=100-\left[\frac{(A 1660 \mathrm{~cm}-1 / \mathrm{A} 3450 \mathrm{~cm}-1) \times 100}{1.33}\right](\%)(2)
$$

Where DD is deacetylation degree; $\mathrm{A}_{1660} \mathrm{~cm}^{-1}$ and $A_{3450} \mathrm{~cm}^{-1}$ are absorbance at $1655 \mathrm{~cm}^{-1}$ and $3460 \mathrm{~cm}^{-1}$ respectively.

The morphological properties of dried nanoparticles were studied using scanning electron microscope (SEM). The SEM having a magnification range of 20000-30000 and accelerating voltage of $15 \mathrm{KV}$ were used for characterization of prepared chitosan and chitosan/gum arabic nanoparticles. All the samples were coated with gold before SEM testing. Similarly, the dimension of the synthesized nanoparticles was measured using Transmission Electron microscopy (TEM) (Philips 400®, $80 \mathrm{KV}$, The 
Netherlands). The samples were immobilized on copper grids and stained with phosphate tungsten acid and examined by TEM.

The invivo test was carried out by acquiring and conditioning the animals for two weeks before the test, using the standard protocol for laboratory animals. Surgical defects (Mandibular defects) were created and managed post operatively by trained personnel in the procedure. Linear submandibular incision was made approximately $10 \mathrm{~mm}$ from the lower border of the mandible using a \#15 blade mounted on a \#3 cable scalpel. Blunt dissection was performed by detaching the masseter muscle and periosteum, following which the flap was retracted with fine retractors. The defect site of each rabbit was filled with the test material allocated to it and appropriately labelled with an indelible marker. For example, the mandibular defect in Rabbit labelled A was filled with gum arabic; Rabbit B was filled with chitosan while Rabbit $\mathrm{C}$ was filled with chitosan/gum arabic nanoparticles. The wound was sutured in layers.

The animals were sacrificed after 21 days using standard protocols. The masseter muscle and periosteum were detached from the bone. The bone tissues were processes in the laboratory and mounted on slides and stained in Haematoxylin and eosin $(\mathrm{H}$ and $\mathrm{E})$ for histological analysis.

\section{Result and Discussion}

\subsection{Results}

The SEM micrographs obtained for both chitosan and chitosan /gum arabic nanoparticles were presented in Plate 1. Similarly, the TEM micrographs for the synthesized chitosan/gum arabic nanoparticles is shown in Plate 2. The FTIR spectra obtained for the chitosan, gum arabic and chitosan/gum arabic nanoparticles were presented in Figure 1 - Figure 3. The result of the wave number and chemical group of FTIR absorption bands and XRF results are shown in Table 1 and Table 2 respectively. The result of the compressive strength, Young's Modulus, \% elongation and yield strength of the synthesized chitosan and chitosan/gum arabic nanoparticles are presented in (Figure 4 a-d). Finally, the In-vivo results, Plate 3, show the photomicrographs interaction of bone with gum arabic, chitosan, chitosan/gum arabic nanoparticles after 21 days of implantation.

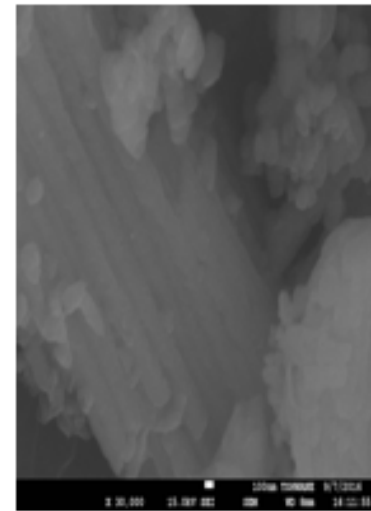

(a)

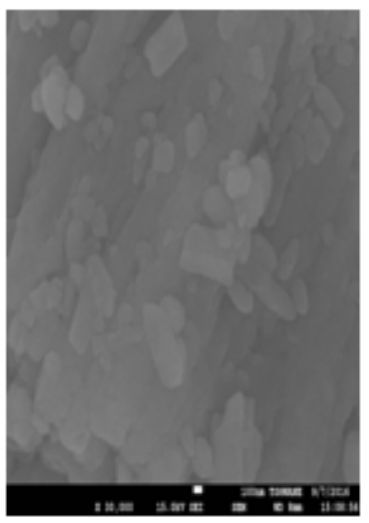

(b)
Plate 1. SEM Micrograph of (a) chitosan (b) chitosan/ gum arabic nanoparticle (Magnification x30000)

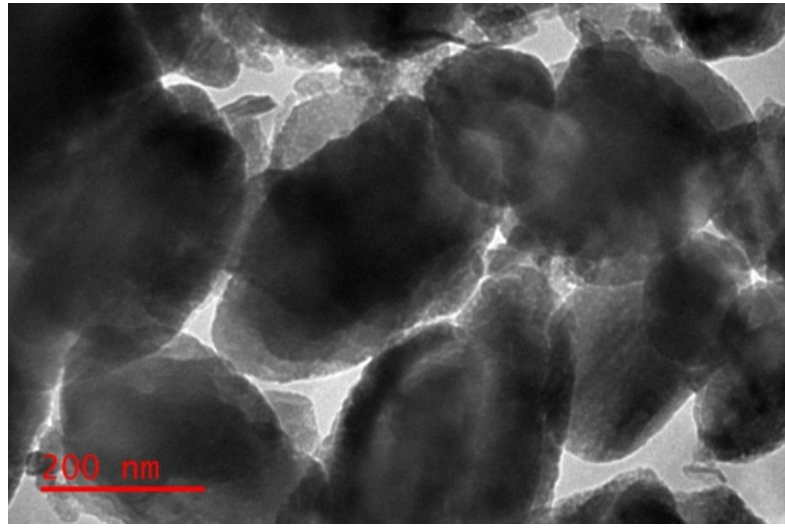

Plate 2. TEM Micrograph of chitosan/gum arabic nanoparticles

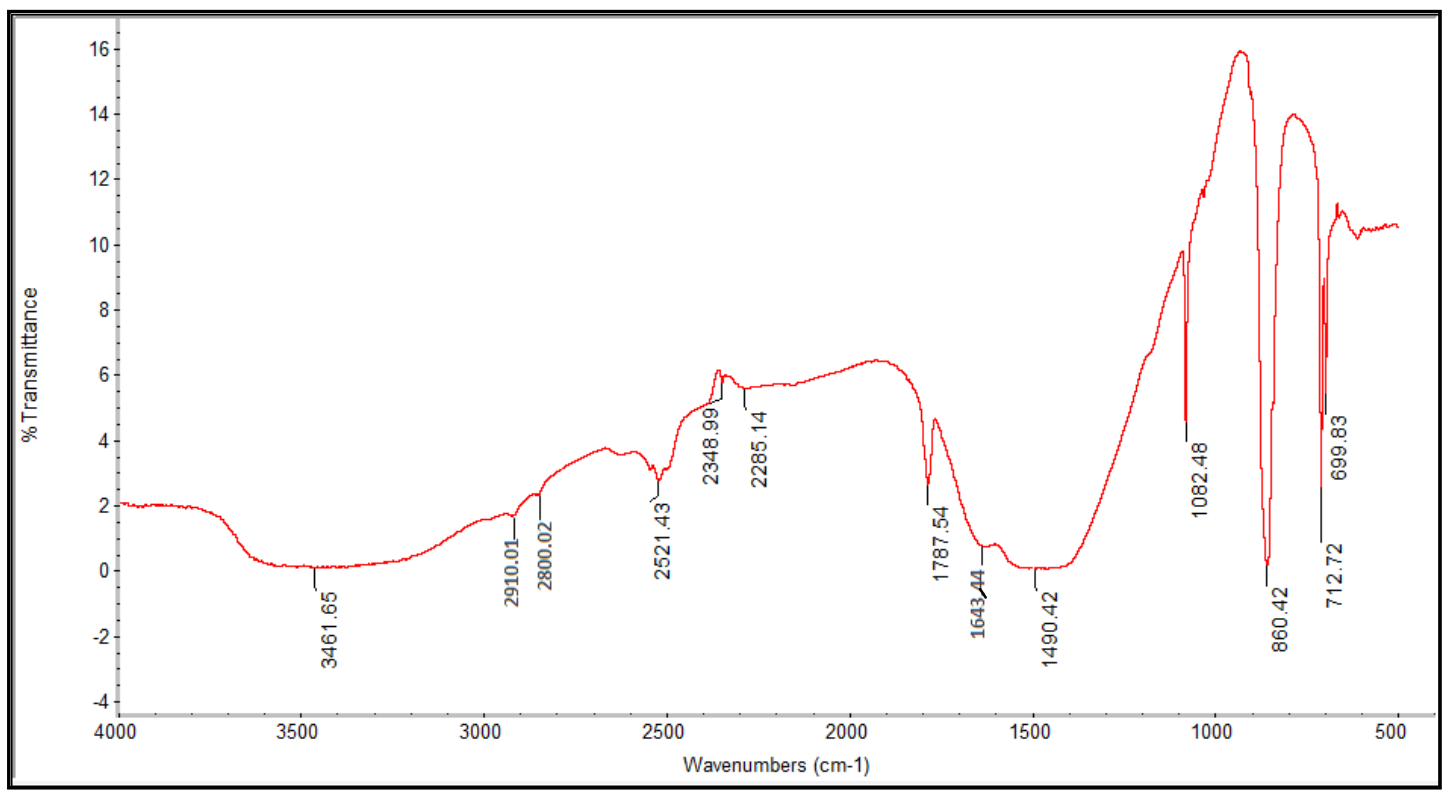

Figure 1. FTIR Spectrum of chitosan 


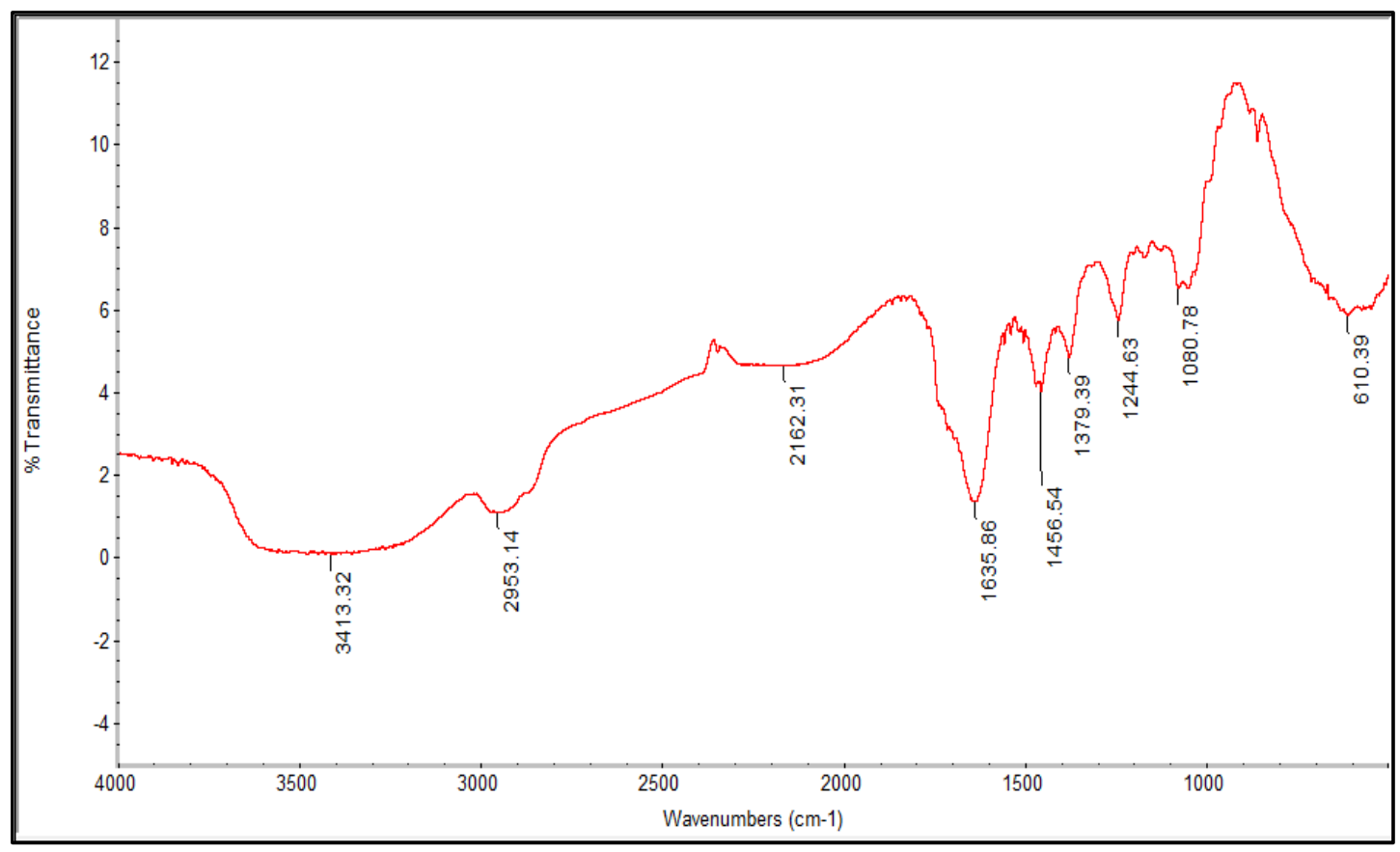

Figure 2. FTIR Spectrum of gum arabic

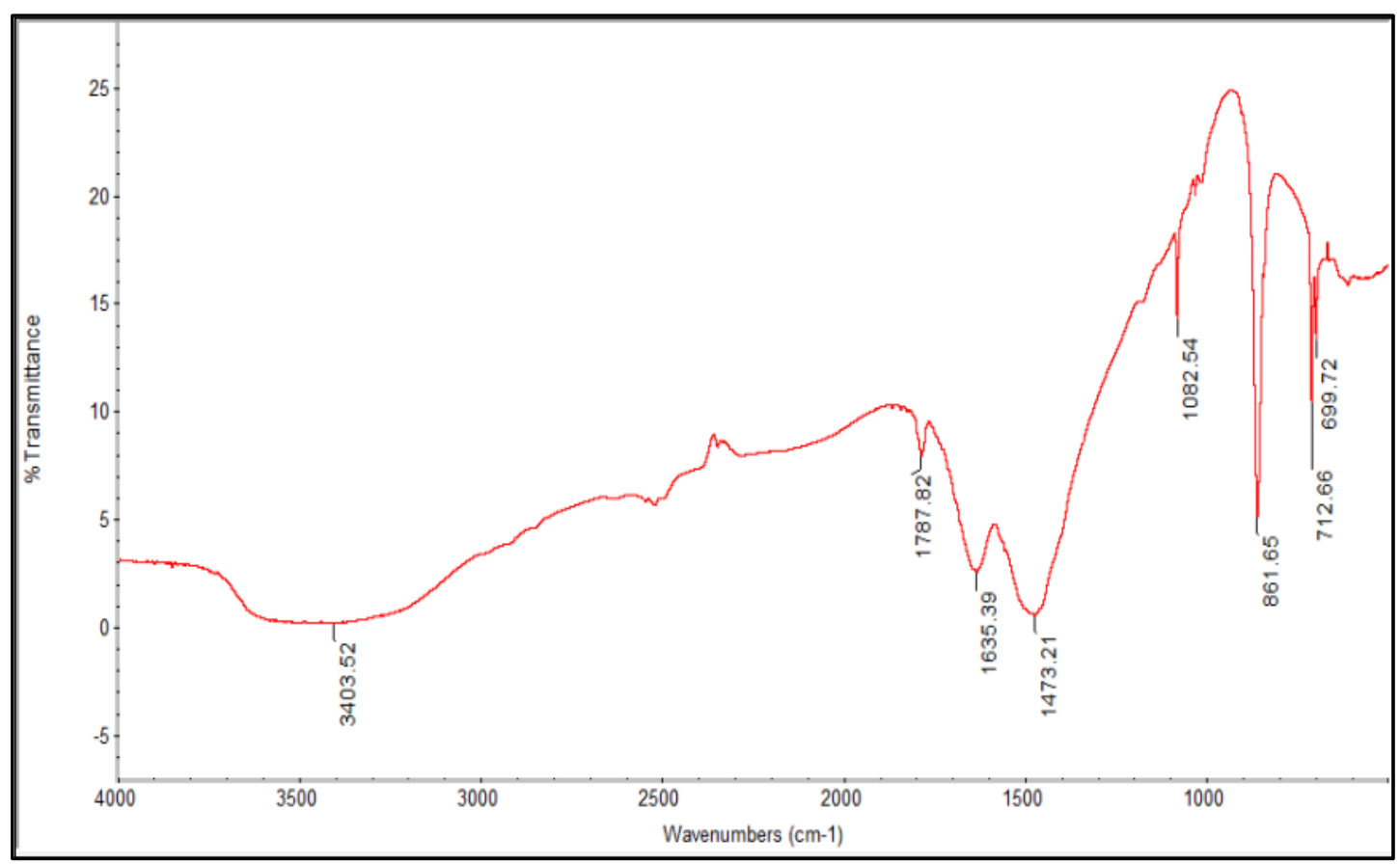

Figure 3. FTIR Spectrum for chitosan/gum arabic nanoparticles

Table 1. Characteristic absorption band of the chitosan and gum arabic

\begin{tabular}{ccl}
\hline Materials & wave length $\left(\mathrm{cm}^{-1}\right)$ & \multicolumn{1}{c}{ Vibration Mode/ Chemical groups } \\
\hline chitosan & $3462-3000$ & $v\left(\mathrm{NH}_{2}\right)$ assoc. in primary amine (OH)assoc. in pyranose hydroxyl group \\
& $2910-2800$ & vas $\left(\mathrm{CH}_{2}\right)$ inCH $\mathrm{CH}_{2} \mathrm{OH}$ group \\
& 1643 & $\mathrm{v}(\mathrm{C}-\mathrm{H})$ in $\mathrm{CH}_{3}$ pyranose ring \\
& 1082 & Amide 1 band $\mathrm{v}(\mathrm{C}=\mathrm{O})$ in $\mathrm{NHCOCH}$ group \\
& 860 & $\mathrm{v}(\mathrm{C}-\mathrm{O})$ in secondary OH group \\
& $\mathrm{CH}_{3} \mathrm{COH}$ group in pyranose ring vibration (Corresponding to saccharide structure ) \\
& \\
Gum arabic & 3413.32 & $\mathrm{O}-\mathrm{H}$ stretching , characteristic of glucosidic ring \\
& 2953.14 & $\mathrm{C}-\mathrm{H}$ stretching \\
& 1635.86 & COO- symmetric stretching \\
& 1456.54 & COO- asymmetric stretching \\
& $1200-900$ & Finger print of carbohydrates \\
\hline
\end{tabular}




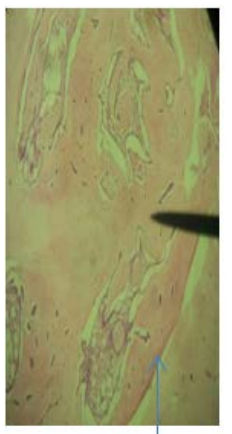

(a)

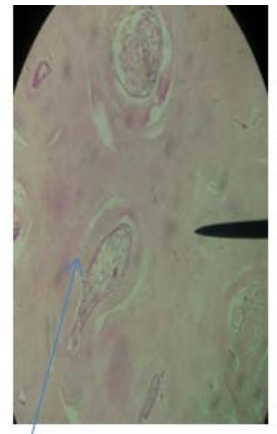

(b)

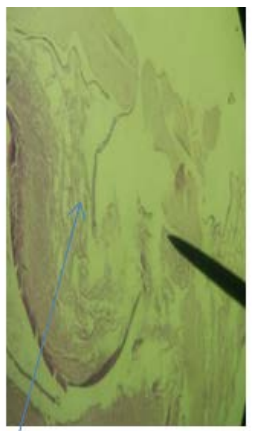

(c)
Plates 3. Photomicrographs of bone at 21 days after implantation of (a)gum arabic; attempts at bone formation(b) chitosan new bone enclosing (c) chitosan/gum arabic nanoparticles (c) bone formation with osteocytes in lacunae present above and beside the test material
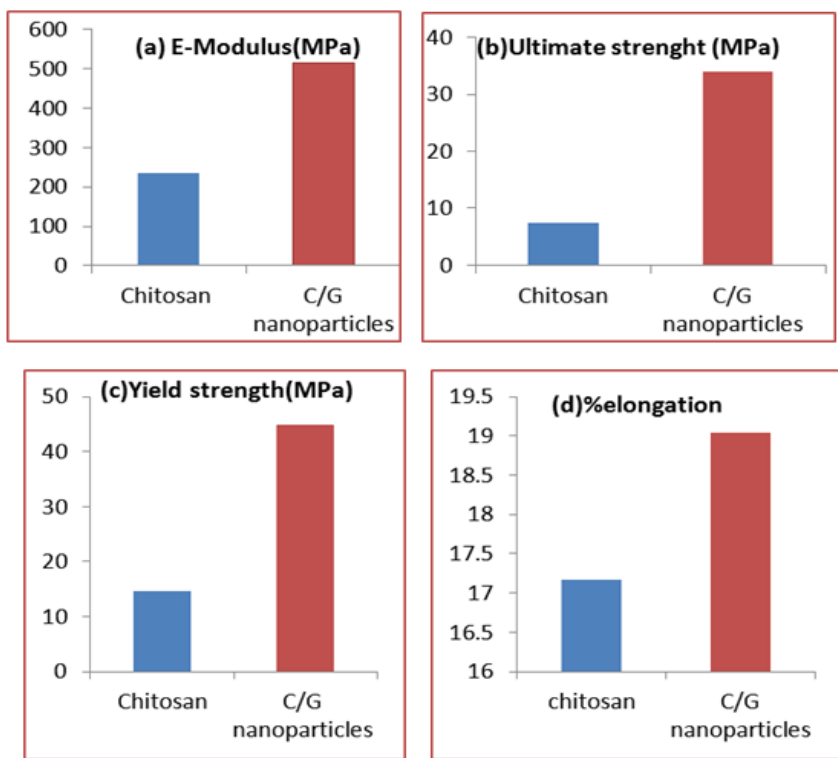

Figure 4. (a-d) The mechanical properties of chitosan and chitosan/gum arabic nanoparticles

Table 2. X-Ray Fluorescence results of chitosan, gum arabic and chitosan/gum arabic nanoparticles

\begin{tabular}{cccc}
\hline element & $\begin{array}{c}\text { Chitosan } \\
\text { (Wt. \%) }\end{array}$ & $\begin{array}{c}\text { Gum arabic } \\
\text { (Wt. \%) }\end{array}$ & $\begin{array}{c}\text { Chitosan /gum arabic } \\
\text { nanoparticles }\end{array}$ \\
\hline $\mathrm{P}$ & 1.7673 & 5.5235 & 1.0798 \\
$\mathrm{Cl}$ & 1.2200 & 3.2972 & 0.9725 \\
$\mathrm{~K}$ & 1.7494 & 8.6066 & 1.7445 \\
$\mathrm{Ca}$ & 35.7397 & 18.2917 & 36.6864 \\
$\mathrm{~V}$ & 0.0213 & 0.0808 & 0.0185 \\
$\mathrm{Mn}$ & 0.0210 & 0.3314 & 0.0113 \\
$\mathrm{Fe}$ & 0.1142 & 1.9781 & 0.1343 \\
$\mathrm{Cu}$ & 0.0156 & 0.5176 & 0.0142 \\
$\mathrm{Zn}$ & 0.0498 & 0.2196 & 0.0204 \\
$\mathrm{Ga}$ & 0.0091 & 0.0781 & 0.0089 \\
$\mathrm{Se}$ & 0.0043 & 0.0909 & 0.0048 \\
$\mathrm{Sr}$ & 0.0349 & 0.0686 & 0.0412 \\
$\mathrm{Ti}$ & 0.0120 & 0.2355 & 0.0191 \\
$\mathrm{Mo}$ & 0.0033 & 0.0916 & 0.0025 \\
$\mathrm{Cr}$ & 0.0039 & 0.1568 & 0.0024 \\
$\mathrm{Ni}$ & 0.0261 & 0.4866 & 0.0295 \\
$\mathrm{As}$ & 0.0024 & 0.0308 & 0.0036 \\
$\mathrm{Bi}$ & 0.0087 & 0.2028 & 0.0087 \\
$\mathrm{~W}$ & 0.0108 & 0.4078 & 0.0106 \\
$\mathrm{~Pb}$ & 0.0006 & 0.0444 & 0.0010 \\
$\mathrm{Nb}$ & 0.0034 & 0.0886 & 0.0039 \\
\hline & & &
\end{tabular}

\subsection{Discussion}

\subsubsection{Mechanical Properties}

Mechanical properties of a scaffold used for tissue engineering are very important due to the need for the structural stability to oppose the various stresses incurred during culture, in-vitro or implantation, in-vivo [24]. Bone tissues undergo compression and due to that compression test was used for evaluation. The result of this study has shown that The Young Modulus (compression) for chitosan and chitosan/gum arabic nanoparticles were 235 and $515 \mathrm{MPa}$ respectively. The result strongly indicated that the Young Modulus (compression) chitosan/gum arabic nanoparticles is higher than native chitosan. Similarly, compressive strength, \% elongation and yields strength shown in Figures 3 indicate that chitosan/gum arabic nanoparticles have a better mechanical properties than chitosan The observed increased in the mechanical strength is ascribed to cross linking of amine group of chitosan with carboxylic acidic of the gum arabic leading to enhance mechanical properties of the chitosan/gum arabic nanoparticles. The Young's Modulus (compression) and the ultimate's strength of bone are at the range of 1-20GPa and 1-200 $\mathrm{MPa}$ respectively [27]. The chitosan/gum arabic nanoparticles synthesized has Young's Modulus (compression) and the ultimate compressive strength of $515 \mathrm{MPa}$ and $34 \mathrm{MPa}$ which are nearer and within the range of human bone respectively. This shows that it will function well as bone graft substitute.

\subsubsection{Morphology Characterisation}

Plate 1 show SEM of chitosan and chitosan/gum arabic nanoparticles. The morphology of chitosan presented a typical of polymeric rods, with smooth surface and they agree with the previous observation by [23], where it was discovered that chitosan conformations depend on molecular weight. When the molecular weights are as high as $223 \mathrm{kDa}$, chitosan will acquire a random coil shape. While, when chitosan molecular weights are low such as below $148 \mathrm{kDa}$, chitosan will form a rod shape structure. Similarly, when the chitosan crossing linking reaction occurred through the addition of gum arabic, aggregated particulates were formed as noted by [24]. Higher hydrogen ion concentration favours rearrangement of protonated chitosan free chains, leading to formation of semi spherical shapes and homogeneous population of nanoparticles. This might be responsible for observed enhanced mechanical properties of chitosan/gum arabic nanoparticles. The TEM analysis confirms that the synthesized chitosan/gum arabic nanoparticles have a mean particle size of $200 \mathrm{~nm}$ which is in the range of biological molecules and much smaller than cells [25] and this will favours bone cells adsorption.

\subsubsection{RF of the Synthesized Materials}

The result X RF of the analysis obtained for chitosan, gum arabic and chitosan/ gum arabic nanoparticles (Table 2) show the purity of the synthesized chitosan and chitosan/gum arabic nanoparticles. It can be noted that the amount of trace elements present in the synthesized materials are very low. This confirms that the synthesized 
products are pure chitosan and chitosan/gum arabic nanoparticles. This implies that the synthesized materials will be suitable for bone regeneration. Evaluation of element with atomic number $(\mathrm{Z})$ less than 11 is however, not possible with this technique. Hence, major elements such as $\mathrm{C}, \mathrm{O}, \mathrm{H}$ and $\mathrm{N}$ were not visible. However, the FTIR results show some of the major functional group present in the synthesized materials.

\subsubsection{FTIR Spectra of the Synthesized Materials}

The FTIR spectrum of chitosan/gum arabic nanoparticles, in Figure 3, shows that few significant changes was occurs in chitosan/gum arabic nanoparticles compared to the native chitosan (Figure 1).

FTIR spectrum of chitosan shows a peak at $3461 \mathrm{~cm}^{-1}$ which indicates symmetric stretching vibration of $\mathrm{O}-\mathrm{H}$ bond (in alcohol) and N-H amine while the absorptions bands observed at $2910 \mathrm{~cm}^{-1}, 2800 \mathrm{~cm}^{-1}, 1643 \mathrm{~cm}^{-1}$ and $1082 \mathrm{~cm}^{-1}$ are respectively attributed to the stretching vibration of $\mathrm{CH}$ (in methylene), $\mathrm{CH}$ (in methyl), $\mathrm{C}=\mathrm{O}$ in (amide 1) and C-O-C in (glucosamine ring). This peaks are similar to the one observed by $[26,27]$. In the chitosan/gum arabic nanoparticles, the tip of the peak $3461 \mathrm{~cm}^{-1}$ on chitosan, has shifted to $3403 \mathrm{~cm}^{-1}$ and becomes wider. This resulted from superimposed $\mathrm{OH}$ and $\mathrm{NH}_{3}{ }^{+}$stretching band [11]. The absorption in $1635 \mathrm{~cm}^{-1}$ and $1473 \mathrm{~cm}^{-1}$ correspond to the presence of asymmetric $\mathrm{N}-\mathrm{H}\left(-\mathrm{NH}_{3}{ }^{+}\right)$band and asymmetric -COO- stretching respectively. Peaks at $1080 \mathrm{~cm}^{-1}, 860 \mathrm{~cm}^{-1}$ and $712 \mathrm{~cm}^{-1}$ in chitosan/gum arabic nanoparticles spectrum were similar to the native chitosan spectrum, which indicates no change in the main backbone of chitosan structure [28]. These resulted ionic interaction and multiple intermolecular hydrogen bonding between the $-\mathrm{NH}_{2}$ groups of chitosan and consequently the crosslinking that resulted in the observed improved mechanical properties [11,28].

\subsubsection{Evaluation of Degree of Deacetylation}

Degree of deacetylation (DD) is an important parameter affecting solubility, chemical reactivity and biodegradability. It depends on the source of the chitosan and procedure used to synthesize it. DD may range from 30-95\%. It is rare that the production of chitosan with $100 \%$ degree of deacetylation is achievable. Therefore, commercial chitosan with various degree of deacetylation in the range of $75-85 \%$ are commonly found [29]. From the FTIR results, using absorbance $A_{3450} \mathrm{~cm}^{-1}$ and $A_{1660} \mathrm{~cm}^{-1}$, the degree of deacetylation (DD) was found to be $70 \%$. This is good anticipated good enough for bone regeneration, as higher DD shows a higher range of cells attachment and higher cellular activities than the lower degree of deacetylation [30].

\subsubsection{In-vivo Analysis}

The results of the in-vivo analysis of the synthesized chitosan/gum arabic nanoparticles, gum arabic and chitosan showed evidence of new bone formation after 21 days post implantation in defect created in the mandibular rami of rabbits (Plates 1-3). All the surgical sites healed with no observed postoperative complications or clinical signs of reaction to any of the materials used in these experiments. Histological analysis of the defect site showed that the defect site implanted with the chitosan /gum arabic nanoparticles displayed some new bone formation after 21 days post implantation (Plates 3c). However, the defect implanted with chitosan particles alone exhibited more bone formation compared to defects treated with chitosan/gum arabic nanoparticles (Plates 3c). In defects treated with gum arabic alone, the bone edges were separated by soft tissue and there was little or no evidence of new bone formation (Plates 3a).

The result of this study is in agreement with previous studies [31,32]. Klokkevold et al. [33] also reported that chitosan increases the activity of osteoblasts and helps bone formation. [34] reported that spongy chitosan supports the proliferation of osteoblastic cells. Several studies have investigated various effects of chitosan on bone healing and raised some hypotheses on its mechanisms [35,36,37]. For instance [38], observerd that chitosan increases the vascularization of blood vessels and stimulates budding tissue (tissue comprising of budding capillaries and fibroblasts) [6,38] noted that the mechanical strength of scaffold decreases with porosity. This might have led to longer healing time of the chitosan/gum arabic nanoparticle in comparison with chitosan. Also, it is important that a scaffold should possess adequate mechanical properties to function effectively at the site of implantation and should be consistent with the anatomical site into which it is implanted. According to Wolfs law, once a new bone is formed, remodeling occur to produce a functional and durable bone tissue such that it stimulates osteoblast and osteoclasts to remodeling bone structure to a better resistance to strain [22,39]. With inadequate strain, induce mode remodeling will result into bone removal. Thus adequate mechanical strains must be present to maintain the newly regenerated bone [39]. Therefore, there is need to balance between mechanical properties and porosity of a scaffold to allow cell infiltration and vascularization.

Furthermore, chitosan/gum arabic nanoparticles (Plate 3a), show the presence of osteoblast, osteocyst and new bone matrix, which indicates progressive new bone formation [40]. Osteoblasts are responsible for synthesizing the organic components of the bone matrix and enzyme alkaline phosphatase, which is needed locally for the mineralization of osteoid, while osteocytes are actively involved with the maintenance of the bony matrix [41]. During bone remodeling, osteoblasts deposit a layer of osteoid seam (approximately $10 \mu \mathrm{m}$ thick) on the surface of the resisting bone, which then begin to mineralize in approximately 20 days. This interval is known as the mineralization lag time [41]. The failure for complete healing of the bone defect within 21 days, is attributed to bone healing been out of the mineralization lag time. Therefore the chitosan/gum arabic nanoparticles has a better mechanical and remodeling ability and subsequently good for bone regeneration.

\section{Conclusion}

Chitosan from cowry shells and chitosan/gum arabic nanoparticles were successfully synthesized for bone regeneration. Mechanical, chemical and microscopic analyses were done to know the suitability of the chitosan 
and chitosan/gum arabic nanoparticles as a bone graft substitute for bone regeneration. SEM micrographs present chitosan and chitosan/gum arabic nanoparticles as a polymeric rods and aggregates respectively while TEM confirms size of the chitosan/gum arabic nanoparticles as $200 \mathrm{~nm}$. FTIR results showed the functional groups in chitosan and chitosan/gum arabic nanoparticles while $\mathrm{XRF}$ was able to show some of the elements present in the synthesis products. The compressive strength, Young Modulus, yield strength and \% elongation were enhance by $79.3 \%, 54.4 \%, 67.5 \%$ and $7 \%$ respectively. In-vivo results were able to show bone regeneration properties of chitosan and chitosan/gum arabic nanoparticles. Chitosan/gum arabic nanoparticles has bone regeneration properties with better mechanical properties. Hence chitosan application as a bone graft substitute is enhanced.

\section{Recommendation}

Based on the findings of the study, it is recommended that longer term histological and histomorphometric studies be carried out to understand better the healing times and the nature of remodeling bone formed by chitosan/gum arabic nanoparticles and chitosan.

\section{Contribution to Knowledge}

Chitosan/gum arabic nanoparticles with mechanical properties comparable to human bone have been developed with potential to serve as bone regeneration scaffold.

\section{References}

[1] Costa-Pinto, A., Reis R., \& Neves N.M., (2011). "Scaffolds Based Bone Tissue Engineering: The Role of Chitosan," Tissue Engineering, Part B, Volume 17, no. 5, Pp.1-18.

[2] Torres, J. Tamimi F., Alkhraist M, Carlos J,. Prados-Frutos and Lopez- Cabarcos (2011). E. Bone Subtitutes, implant DentistryThe most promising Discipline of Dentistry, prof. IIser Turkyilmaz (Ed.), InTech, Vol. 4 Pp. 91-108.

[3] Polo-Corrales, L., Latorre-Esteves, M., and Ramirez-Vick, J. (2014). "Scaffold Design for Bone Regeneration," J Nanosci Nanotechnol, Vol. 14, no. 1, Pp. 15-56.

[4] Dimitriou, R., Jones, E., McGonagle, D. and Giannoudis, P. (2011). "Bone Regeneration: Current Concepts and Future Directions,” BMC medicine, Vol. 9, no. 66, Pp.1-10.

[5] Sandor, G., Lindholm, T. and Clokie, C., (2003). Bone Regeneration of the Craniomaxillofacial and Dento-alveolar Skeletons in Framework of Tissue Engineering. Topic on Tissue Engineering (2003): Ashammakhi, N., and Ferretti, P., eds university of Oulu, (2003) chapter 7, Pp. 1-46.

[6] O'Brien, F. (2011). "Biomaterials and scaffolds for Tissue Engineering,” Material today, Vol. 14, Pp. 88-95.

[7] Motamedia, S. Hosseinpour, S. Ahsale M., and Khojasteh, A. (2015). "Smart scaffolds in bone tissue engineering: A systematic review literature. worlde J stem Cells, Vol. 7, no. 3, Pp. 657-668.

[8] Rodriguez-vazquez, M., Vega-Ruiz, B., Ramos-Zuniga, R., Saldana-Koppel, D. A., and Quinones-Olvera, L. F., (2015). "Chitosan and Its Potential Use as a Scaffold for Tissue Engineering in Regenerative Medicine" BioMed Research International, Vol. 2015, Pp. 1-15.

[9] Mota, J., Yu N., Caridade S. G., Luz, G. M., Gomes E. M., Reis R. L., Jansen J. A., Walboomers X. F., Mano J. F. (2012). "Chitosan/bioactive Glass Nanoparticle composite membranes for periodontal regeneration” Acta Biomaterialia, Vol. 8, Pp 4173-4180.
[10] Ahmed, S. and Ikram, S. (2016). “Chitosan Based Scaffolds and Their Applications in Wound Healing." Achievements in the Life Science, Vol. 10, Pp. 27-37.

[11] Sailakshmi, G. Mitra, T., Chatterjee, S. and Gnanamani A. (2013). "Engineering Chitosan using $\alpha, \dot{\omega}$ Dicarboxylic acids-An approach to improve mechanical strength and thermal stability,” Journal of Biomaterials and Nanobiotechnology, no. 4, Pp. 151-164.

[12] Montenegro, M. A., Boieroi, Valle L. and Borsarelli C.D., (2012): "Gum arabic more than an Edible Emulsifier, products and Applications of Biopolymers,” Dr. Johan Verbeek (Ed.), InTech, Available from: http://www.intechopen.com/books/products-Pp. 3-27.

[13] Leloni, J. K., Jumba I.O., Keter J.K., Chemuku W. and Oduor F.D.O. (2010). Assessment of Physical Properties of Gum Arabic from Acacia Senegal Varieties in Baringo District, Kenya.

[14] Azeez, O. S. (2005). "Decolourization of Gum Arabic Using Activated Charcoal”. Leonardo Journal of Sciences, Pp. 23-32.

[15] Avadi, M.R., Sadeghi, M. M., Dounighi, N. M., Dinarand, R., Atyabi , F. and Rafiee-Tehrani, M., (2011). "Ex Vivo Evaluation of Insulin Nanoparticles using Chitosan and Gum Arabic.” International Scholarly Research, Vol. 2011, Pp.1- 6.

[16] Zhao, L., Shi, L. Z., Chen, J., Shi, D., Yang, J. and Tang, Z. (2011). "Preparation and Application of Chitosan Nanoparticles and Nano fibers,” Brazilian Journal of chemical Engineering, Vol. 28, No. 03, Pp. 353-362.

[17] Lanka D. and Mittapally (2016). "Preparation and Application of Chitosan Nanoparticles: A Brief,” Research and Reviews: Journal of Material Science, Pp. 1-5.

[18] Anil, S., Al-Sulaimani, A.F., Beeran, A. E., Chalisserry, E. P., Varma, P. R. and Al-Amri, M. D. (2015). Drug Delivery Systems in Bone Regeneration and Implant Dentistry. Current concept in dental implantology, King Saud University, Riyadh, Saudi Arabic INTECH Chapter 10: Pp.239-264.

[19] Sailaja, A., Amareshwar, P. and Chakravarty, P. (2010). Different Techniques used for the Preparation of Nanoparticles using Natural Polymers and their Application. International Journal of pharmaceutical science, Vol. 3, no. 2, page 1-8.

[20] Kafshgari W. H, Khorram W., Khodoost W. and Khavari S. (2011). "Reinforcement of Chitosan Nanoparticles Obtained by an Ionic cross-linking process,” Iranian Polymer Journal, Pp 445-456.

[21] Ikeda, T., Ikeda, K., Yamamoto, K. Ishizaki, H. Yoshizawa Y., Yanagiguchi, K. Yamada, S. and Hayashi, (2014). "Fabrication and Characteristic of Chitosan Sponge as a Tissue Scaffold" BioMed Research International volume, 2014 (2014), Pp. 1-8.

[22] Yildirim, O. (2004). "Preparation and Characterization of Chitosan/ Calcium Phosphate based Composite Biomaterials”. Masters Research Work, Master of Science and Engineering, Institute of Technology Zmir, Turkey.

[23] Al-Remawi, M., (2012). "Properties of Chitosan Nanoparticles Formed Using Sulphate Anions as Crosslinking Bridges,” American Journal of Applied Science, Vol. 9, no. 7, Pp. 1091-1100.

[24] Esquivel, R., Juarez, Almada, M., Ibarra, J. and Valdez, M. A. (2015). "Synthesis and Characterization of New Thiolated Chitosan Nanoparticles Obtained by Ionic Gelation Method," International Journal of polymer Science, Vol. 2015, Pp. 1-18.

[25] Kemp, M. M. and Linhardt, R. J. (2010). Heparin-based Nanoparticles. John Wiley and Sons, Inc, Vol. 2, Pp. 77-87.

[26] Zvezdova D., (2010). "Synthesis and characterization of chitosan from marine sources in Black sea," НАУЧНИ ТРУДОВЕ НА РУСЕНСКИЯ УНИВЕРСИТЕТ - 2010, том 49, серия 9.1, Pp. 65-69.

[27] Puvvada, Y., Vankayalapati, S. and Sukhavasi, S. (2012). "Extracion of Chitin from Exoskeleton of Shrimp for Application in the Pharmaceutical Industry," International Current Pharmaceutical Journal, Vol. 1, no. 9, Pp. 258-263.

[28] Mitra, T., Sailakshmi, G. Gnanamni, A. and Mandal, A. B. (2013). "Studies on Cross-linking of Succinic Acid with Chitosan/Collagen," Material Research, Vol. 16, no. 4, Pp. 755-765.

[29] Islam, M., Masum, S., Rahman, M., Molla, I., Shaikh, A. and Roy, S. K. (2011). "Preparation Of Chitosan from Shrimp Shell and Investigation of Its Properties” International Journal of Basic and Applied Science, Vol. 11, no. 01, Pp. 77-80.

[30] Nwe, N., Furuike, T. and Tamura, H. (2009). “The Mechanical and Biological Properties of Chitosan Scaffolds for Tissue 
Regeneration Templates are Significantly Enhanced by Chitosan," Materials, Vol. 2009, no. 2, Pp. 374-398.

[31] Cui, X., Zhang, B., Wang, Y. and Gao, Y. (2008). "Effects Of Chitosan-coated Pressed Calciumsulfate Pellet Combined with Recombinant Human Bone Morphogenetic Protein 2 On Restoration of Segmental Bone Defect," Journal of Craniofacial Surgery, Vol. 19, Pp. 459-465.

[32] Lee, K.Y., Ha, W.S. and Park, W.H. (1995). "Blood Compatibility and Biodegradability of Partially N-acylated Chitosan Derivatives," Biomaterials, Vol. 16, Pp. 1211-1216.

[33] Klokkevold, P.R and Newman, M.G (2000). "Current Status of Dental Implants: A periodontal perspective," International Journal of Oral and Maxillofacial Implants, Vol. 15, Pp. 55-65.

[34] Lee, Y.M, Park, Y.J., Lee, S.J., Ku, Y., Han, S,B., Choi, S.M., et al (2000). "Tissue Engineered Bone Formation Using Chitosan/tricalcium Phosphate Sponges" Journal of Periodontology, Vol. 71, Pp. 410-417.

[35] Mizuno, K., Yamamura, K., Yano, K., Osada, T., Saeki, S., Takimoto, N. (2003). "Effect of chitosan film containing basic fibroblast growth factor on wound healing in genetically diabetic mice,” Journal of Biomedical Materials Research, Part A, no. 64, Pp. 177-181.
[36] Ueno, H., Murakami, M., Okumura, M., Kadosawa, T., Uede, T. and Fujinaga, T. (2001). "Chitosan accelerates the production of osteopontin from polymorphonuclear leukocyte,"] Biomaterials, Vol. 22, Pp. 1667-1673.

[37] Ueno, H., Nakamura, F., Murakami, M., Okumura, M., Kadosawa, T. and Fujinag, T. (2001). "Evaluation Effects of Chitosan for the Extracellular Matrix Production by Fibroblasts and the Growth Factors Production by Macrophages," Biomaterials, Vol. 22, Pp. 2125-2130.

[38] Chevrier, A., Hoemann, C.D., Sun, J. and Buschmann, M.D. (2007). "Chitosanglycerol Phosphate/blood Implants Increase Cell Recruitment, Transient Vascularization and Subchondral Bone Remodeling in Drilled Cartilage Defects," Osteoarthritis Cartilage, Vol. 15, Pp. 316-327.

[39] Luo, J., Sun, M., Kang, Q., Peng, Y., Jiang, W., Luu, H.H., Luo, Q., Park, J., Li, Y., Haydon, R.C., Hei, T. (2005). “Gene Therapy for Bone Regeneration”, Current Gene therapy, Vol. 5, Pp. 167-179.

[40] Landsman, R. and Reddi, A. H., (1986). "Chemotaxis of Muscle Derived Mesenchymal Cells o Bone-Inductive Proteins of Rat," Calcified Tissue International, Vol. 39, Pp. 259-262.

[41] Roza G., (2016). “Histology of Bone,” Gellman H., ed. Medscape Pp1-9. 